\title{
La folie de Lear ou de l'inceste alimentaire
}

\section{Ann Lecercle}

\section{Q OpenEdition \\ Journals}

\section{Édition électronique}

URL : http://journals.openedition.org/shakespeare/1311

DOI : 10.4000/shakespeare.1311

ISSN : 2271-6424

Éditeur

Société Française Shakespeare

Édition imprimée

Date de publication : 1 novembre 1989

Pagination : 43-58

\section{Référence électronique}

Ann Lecercle, "La folie de Lear ou de l'inceste alimentaire », Actes des congrès de la Société française Shakespeare [En ligne], 7 | 1989, mis en ligne le 01 janvier 2007, consulté le 19 avril 2019. URL : http:// journals.openedition.org/shakespeare/1311; DOI : 10.4000/shakespeare.1311 


\title{
Société Française Shakespeare
}

\author{
Actes des Congrès \\ 1985 - 1986 - 1987
}

\section{LA FOLIE}

\section{SHAKESPEARE ET LES ARTS \\ LE TRAGIQUE}




\section{LA FOLIE DE LEAR OU DE L'INCESTE ALIMENTAIRE}

\section{Ann LECERCLE}

Dans l'un des premiers textes «théoriques» de l'époque élisabéthaine, Sir Philip Sidney déploie un argument aussi admirablement conçu qu'exprimé. Le noyau en est cette imbrication fondamentale entre le verbal et l'iconique, entre la parole et quelque chose qui dépasse parce qu'elle précède la parole, parce qu'elle est d'avant la parole, et que Sidney appelle myth. Pour lui en effet la poésie est «a speaking picture» a perfect picture [...] not a wordish description [...] which doth neither strike, pierce, nor possess the sight of the soul so much as the other doth. $[. . .]^{1}$

For, as Aristotle saith, it is not gnosis, but praxis must be the fruit. And how praxis cannot be, without being moved to practise, it is no hard matter to consider ${ }^{2}$.

C'est que, s'il veut «Hold children from play, and old men from the chimney corner», le poète doit prendre son lecteur/spectateur non pas seulement par le savoir mais par le plaisir, et partant, par le désir, d'où le passage chez Sidney de entice mot fort - in titio, (la torche qui met le feu), à taste :

For the poet doth not only show the way, but giveth so sweet a prospect into the way, as will entice any man to enter into it. Nay, he doth, as if your journey should lie through a fair vineyard, at the first give you a cluster of grapes, that, full of taste, you may pass furthern'.

Voilà, dites-vous, des idées bien connues. Sans doute. Mais la critique est loin d'en tirer toutes les conclusions qui s'imposent : à savoir qu'il faut saisir l'œuvre 
d'art là où elle nous «empoigne», pour parler comme Freud, dont le raisonnement dans Le Moïse de Michel-Ange est identique dans le fond, sinon dans la forme, à celui de Sidney. Reconstituer un savoir, soit, mais aussi cette imbrication entre le verbe et l'icône, entre récit et représentation, qui éveille le désir et crée le plaisir et s'appelle pour Sidney myth, pour Freud fantasme. A fortiori là où il s'agit de la folie, et notamment quand celle-ci s'apparente à la folie tyrannique. Ici, c'est Socrate, qui a posé, une fois pour toutes, les prolégomènes à son analyse dès les premiers mots du $4 \mathrm{e}$ livre de La République :

Attends! sais-tu bien, dis-je, ce que je réclame encore? Quoi ? La question des désirs, de leur nature et de leur nombre, n'a pas eté, d mon avis, convenablement analysée par nous : une insuffisance sur ce point obscurcira évidemment la recherche a laquelle nous nous livrons.

Ces mots de Socrate, nous les mettons en exergue à nos remarques sur Lear. Car enfin, on est bien obligé, avant d'entrer en matière, d'évoquer la formidable résistance qui se fait jour dans le corpus critique consacré à cette pièce. Si, d'une part, on y trouve mainte page éloquente sur la «violence», «l'agressivité» et autres notions analogues, qui atteignent, nous dit-on, un degré unique dans Shakespeare avec cette pièce - ce que l'on résume habituellement par le terme de "primitivisme» - d'autre part, force est de constater, au-delà de ce consensus superficiel, l'existence d'une espèce de continent noir qui subsiste au centre même de l'œuvre et qui semblerait relever de l'ineffable. Ce qui se traduit le plus souvent par une débauche de métaphores, «mixtes» le plus souvent, de circonlocutions en tout genre, et par toute la batterie conradienne des épithètes de l'indicible, qui masque pourtant mal le manque d'articulation de ce précisément en quoi consiste cette «irrationalité». Ceci donne, chez Wilson Knight, qui remporte la palme du purple passage : «[it is an irrationality] touching some chord of primitive mentality, some stratus in subconsciousness reaching back aeons of the evolutionary process, now tumbled up in the loosened activity of madness». Hélas, ce qui est «tumbled up» ici, c'est l'activité du critique, non celle de la folie. Car enfin, l'Enfer comme le Paradis, l'irrationnel comme le rationnel, l'inconscient comme le conscient, se structurent. Ce qu'il faut montrer, c'est que cet ineffable est de l'ordre de l'impensé, non de l'impensable. Mieux, cet ineffable se dit, se dit à la lettre et dans la lettre. 
Au-delà des oppositions de surface (bonne fille/mauvaise fille), la scène d'ouverture, dès les premiers mots, oppose d'emblée les deux faces de l'appétit. Dans ce qui se présente comme un court prologue à l'action principale (I, i, I-33), il s'agit d'établir l'appétit sexuel, génital, du Duc de Gloucester, caractérisé tout au long de l'œuvre par la démesure de l'Eros. Cet être, le premier que voit le public, c'est le lecher de la pièce, terme dont les origines orales (lécher) sont transparentes. D'ailleurs, le personnage met à nu lui-même ce que cette étymologie comporte d'ambivalence pulsionnelle quand il se décrit comme un homme qui se «repaît de luxure», «a lust-dieted man». Ainsi, pour bien préparer son terrain, Shakespeare consacre ce prologue à l'évocation de la conception «savoureuse» (saucy) du fils illégitime du duc, les racines culinaires de l'épithète se passent de commentaire.

Or, si, dans son exploration des impasses et des interdits du désir, King Lear remonte bien au-delà de la tragédie de Sophocle, c'est pour une raison bien précise : dans Lear, ce n'est pas d'inceste qu'il s'agit, mais spécifiquement de ce que Claude Lévi-Strauss appelle «inceste alimentaire». Edith Sitwell avait déjà signalé ce que la pièce de Shakespeare comporte de réécriture de celle de Sophocle. Mais son constat est insuffisant. La problématique shakespearienne se situe à un tout autre niveau. Autrement dit; à travers tous ses avatars - du plus sublimé (le motif traditionnel de la prova d' amore de la première scène) au plus archaïque, le fantasme de festin cannibal sur lequel celle-ci ne manque de déboucher - c'est bien le cas de le dire - c'est bien tout le domaine de l'oralité qui fournit à Shakespeare ce que l'on pourrait appeler la «sustantificque mouelle» de son cuvre (Rabelais, d'ailleurs, n'est pas loin, comme nous le verrons). Et c'est là qu'intervient la folie. Car, si Gloucester, comme EEdipe, a les yeux crevés, Lear, lui, est fou. Or, en dépit de ce que prétend Mr. Sholom J. Kahn, qui écrit : «there has not been enough close attention paid to the question of the precise moment, if any, at which we may say Lear is fully mad», la folie de Lear n'est pas a bolt out of the blue. D'entrée de jeu, le texte de Shakespeare est aussi remarquable par sa précision que par sa cohérence quant à l'étiologie de ce qui, par la suite, revêt des formes plus conventionnelles, comme ia couronne de fleurs portée dans ses cheveux. Point n'est besoin en effet d'inventer des tyrannies passées, comme Kurosawa, ni d'invoquer «a loosening» ou a «tumbling up», comme Wilson Knight : le texte de Shakespeare au contraire se resserre, redouble de rigueur dans son enchaînement, non dans le passé de l'histoire, mais dans le présent du myth/fantasme.

Par conséquent, à l'appétit de Gloucester, dont l'ambivalence est explicite dans le texte, vient s'opposer, dans la partie centrale de cette première scène, l'appétit proprement dit, tout aussi ambigu. En effet, après les discours de cérémonie et de circonstance, quand la demande ne rencontre chez Cordelia que le silence, les masques hiératiques tout à coup tombent : s'enchaînent alors les quatre moments d'un dérapage fulgurant qui initie par ailleurs toute psychanalyse : silence, frustration, agression, régression. C'est alors que surgit l'appétit, non seulement, comme chez Gloucester, marqué par la démesure, mais transgression orale par excellence : «The barbarous Scythian, / Or he that makes his generation messes / To gorge his 
appetite, shall to my bosom / Be as well neighbour'd, pitied, and reliev'd, / As thou my sometime daughter». Derrière cette imprécation, qui est comme le sceau que Lear appose à l'acte de bannissement de Cordelia, c'est la figure de Cronos, paradigme de l'infanticide cannibale, que l'on voit présider à la très conventionnelle prova d'amore. Ainsi, à l'oralité sublimée (demande de parole) se substitue une oralité primaire qui a pour spécificité d'ériger la parole en fétiche, c'est-à-dire en leurre, où le désir se laisse d'autant plus facilement piéger que le manque qu'il recouvre se présente par définition sous les espèces d'une plénitude hyperbolique qui ne fait que refléter en miroir, ce que le désir du sujet a d'excessif. Ce nœud explique pourquoi, dans la dernière scène de la pièce, cette représentation est symétriquement inversée : là, le miroir est physiquement présent sur scène, mais cette fois, le désir, loin d'y rencontrer le fétiche, ne retrouve que le manque, manque pas seulement de paroles, mais encore de l'objet oral le plus pur, le plus désincarné, le plus éloigné de «the mess offlesh», c'est-à-dire le souffle, représentation de l'âme. Ce sont les deux faces d'un même diptyque.

Ce n'est pas non plus un hasard si l'ambivalence pulsionnelle de la prova d'amore se reflète - justement en miroir - dans la double nature des accusations portées par Goneril contre son père : ici aussi, le texte de Shakespeare est aussi précis que rigoureusement logique, ce que la symétrie des vers ne fait que souligner. Ses griefs sont deux, et pas n'importe lesquels, l'épicurisme et la luxure : «epicurism and lust / Makes this our court more like a tavern or a brothels?.

Pourtant, ce qui est capital, ce n'est pas que le paroxysme de colère ait lieu. Ce qui l'est, c'est la nature du fantasme qui le provoque, fantasme dont la force fait aussitôt basculer dans le contraire la représentation qui s'y fait jour. Ce renversement dans le contraire, qui est le moteur même de tout fantasme, Shakespeare l'a ici mis en paroles bien avant que Mélanie Klein ${ }^{8}$ ne l'ait entendu. En effet l'image du Titan, de l'ogre archétypal, qui se repaît de la chair de ses enfants, bascule en l'espace de deux lignes vers le spectacle idyllique du nourrisson pendu au sein de sa mère, qui lui aussi, à sa manière «gorges his appetite» - en consommant le lait de celle-ci, non le sang de ses enfants : «I lov'd her most, and thought to set my rest / On her kind nursery». Comme ce fut le cas de lecher et de saucily, le drive pulsionnel fait dériver le sens figuré vers le sens propre, la forte coloration alimentaire du contexte rendant plus «élémentaire» le sens de nursery. Ce qui pourrait très convenablement se traduire par ce que le fou comique de Jodelle, L'Eugène (1552) - sans conteste le premier fou du théâtre français du 16e siècle - en désignant le but de son existence monstrueusement narcissique, appelle sans ambages «les plaisirs nourriciers de nous»'. Le parallèle n'est pas sans intérêt. Loin de se dépouiller prématurément de ses biens, le fou burlesque de Jodelle compte bien les garder par devers lui outretombe, ou au moins les épuiser ici bas, «Biens, ie dy, que iamais n' acquirent / Les parens qui naistre me feirent, / Et qui ainsy donnez me sont / Qu'd mes héritiers ne revont, / Ains pour rendre ma seule vie / En ses délices assouvis» (I, i). En bouffant tous ses biens, l'Eugène va affamer ses enfants ; en s'en privant, Lear va gaver les siens, même si ce n'est que pour mieux les bouffer ensuite, après tout, la sauce, 
comme le ragoût, n'en sera que plus onctueuse. Bref, si stratégie et transgression sont inverses chez l'Eugène, la logique consciente moins retorse que chez Lear, celle inconsciente moins archaïque - en un mot, la boulimie jusqu'à l'éclatement plutôt que l'anorexie jusqu'à l'inanition - le but reste le même : les délices de la nursery. Ecartelées pour ainsi dire de chaque côté de la Manche, ces deux représentations majeures et complémentaires de la folie au 16e siècle constituent un véritable diptyque, tant sur le plan du genre thêatral que sur celui de la pulsion: voilà en effet les deux faces active/passive, sadique/masochiste de la pulsion orale dans tout ce qu'elle a à la fois de plus féroce et de plus dérisoire. Il y a là un nœud fondamental.

Cette analyse de la première scène permet de situer ce qu'on peut appeler sans abus ce «noyau pathogene» de la folie dans le système nosographique du 16e siècle. Le tableau imaginaire que nous venons de commenter représente en effet non seulement l'étiage extrême du renversment dans le contraire propre au fantasme en tant que tel, mais aussi la réalisation d' une coïncidence des contraires qui, en quelque sorte, suture le grand clivage établi à l'époque entre les deux types d'inceste alimentaire : d'une part, la rage canine, incarnée - c'est le cas de le dire - par la figure, paradigmatique depuis Hérodote, Solin et Poponius Mela, du Scythe nordique, et, de l'autre, la fureur cannibale, associée de manière privilégiée aux Tupis des Tropiques. D'un côté, le Scythe bestial (et bête), qui, plus proche du loup que de l'homme, mange de la chair humaine crue, en deça de toute symbolisation, de tout sectionnement, de toute cuisson : de l'autre, l'être essentiellement mélancolique et fourbe, qui, dans un but de vengeance, opère des coupures selon rituel ou recette, que ce soit de simple préparation culinaire ou de dépeçage quasi juridique de l'ennemi. Ces deux extrémités, à la fois géographiques et humorales - suivant par exemple le Methodus ou le livre 5 des Six Bookes of the Commonwealth de Bodin - à savoir, le nord et le sud, respectivement, l'excès de sang et de bile noire, "adust" d'autant plus que l'intensité des rayons du soleil méridional est forte, antinomies, normalement séparées, entre le trentième et le soixantième parallèle, par la région plus modérée de la colère, (de la bile jaune, amère), ces pôles nosographiques, dans cette scène liminaire de Shakespeare, se rencontrent. Or, «l' appétit de vengeance du Méridional ne saurait nullement se confondre avec la rage canine du Scythe ${ }^{10}$; «La fureur se [...] distingue de la rage (il est rare [...] qu' un auteur confonde les deux termes $)^{11}$. Si, ensuite, la folie de Lear éclate en un délire aussi cataclysmique, qui embrase l'univers entier, c'est que, dès le départ, les antipodes planétaires et alimentaires - la rage canine et la fureur cannibale - se télescopent. Dans la bouche de Lear, où les mots «gorge » et «mess» témoignentà fleur de texte d'un débordement en profondeur qui va jusqu'à balayer tous les paramètres différentiels, l'image d'un Scythe vengeur et consommateur de ragoûts incestueux, réunit les deux en une seule et même représentation, la faim à l'état brut et le cannibalisme vengeur, le cru et le cuit. Le sang et le lait aussi. En effet le renversement du fantasme oral dans Lear reproduit en sens inverse le circuit alimentaire, monstrueux parce que fermé, qu'accomplit la femme «allouvie» des Tragiques de D'Aubigné, dévoreuse de son propre enfant : 
Ton sang retournera ou th as pris le laict, Au sein qui t' alloictoit r' entre contre nature ; Ce sein qui t'a nourri sera ta sépulture ${ }^{12}$.

Or, c'est cette représentation clivée, inverse et complémentaire, de ce que Lacan appelleraitl'objetpetita - sang enfantin/lait maternel -qui explique l'emblématisation schématique mais néanmoins précise - et sans doute le choix même - des deux prétendants à la main de Cordelia. Car là aussi, en miroir, c'est le rouge et le blanc qui ressurgissent, pour constituer leur différence. Ce n'est pas un hasard, loin de là, si les deux êtres qui prétendent jouir du corps de Cordelia se présentent dans la bouche de Lear comme «the vines of France and the milk of Burgundy». Ce n'est pas un hasard non plus si Shakespeare répète l'ordre : le vin/sang d'abord, le lait ensuite. Il y a peut-être des questions de préséance, mais c'est mutiler la profonde cohérence du texte shakespearien que de s'en tenir à cette lecture par trop «manifeste».

Il faut ajouter enfin que le vers unique qui sert de «gond» entre les deux volets de ce diptyque oral - spectacle du festin cannibale à gauche, délices de la nursery à droite - «Come not between the dragon and his wrath», lancé à Kent qui tente de s'interposer, est remarquable en ce qu'il constitue à lui seul une représentation par la parole de la transformation qui fait d'un tout-puissant (Cronos) un tout petit, impuissant (nourrisson, ou encore Cronos châtré par Zeus). C'est une dénégation exemplaire, car c'est le roi lui-même qui, dans la formulation de son énoncé, effectue le détachement de la partie sine qua non du tout : la fureur, du dragon. De cette manière il se/le dépouille de son attribut constitutif, emblème de son pouvoir. D'ailleurs, dans le choix même du dragon, c'estl'oralité qui triomphe du phallus, car c'est par la bouche que sort la flamme destructrice. Ce dragon, qui a pour nom Lear, se sert ici de cet organe comme un instrument non de phlogistique mais de linguistique, pour provoquer non une conflagration mais une castration : $c$ 'est très exactement ainsi que l'ogre se retrouve en un clin d'œil mué en nourrisson.

Ainsi s'articulent les deux tableaux - les deux faces - de la problématique orale : être mangé (par le parent), face rouge, sanguinaire/manger (le parent), face blanche, lactée. Au commencement était le Verbe : in the beginning of Lear was the verb. Que l'objet de ce fantasme fondateur de dévoration soit sujet de controverse critique (generation - «make his generation messes» - progéniture et/ou progéniteurs) n'en est que plus symptomatique. L'existence de l'interprétation minoritaire (la seconde, celle de Craig) est à cet égard moins l'indice d'une contradiction que de l'ambivalence radicale de la situation duelle originaire, sans autre issue que la réciprocité la plus absolue. Ce brouillage, pour ne pas dire cette négation des générations se retrouve dans la stratégie de l'Eugène : n'ayant rien hérité de ses parents ${ }^{13}$, n'allant rien non plus transmettre à ses héritiers, il sera, si ce n'est que par métonymie - les biens pour la semence - sans antécédents ni descendants : en un mot, l'incarnation comique d'un Coriolan «author of himself», ou d'un Lear, ogre 
ou nourrisson, consommateur en aval ou en amont de sa génération.

Or, cette situation de brouillage des limites est justement celle qui est évoquée par Albany, en plein déchaînement des atrocités, dans un discours à tonalité nettement chorique, qui, dans le contexte du Roi Lear, est unique de son espèce, unique aussi par le vers de seulement trois pieds qui annonce l'énoncé central : «If that the heavens do not their visible spirits / Send quickly down to tame these vilde offences, / It will come, / Humanity must perforce prey on itself, / Like monsters of the deep». En clair, le macrocosme entier est envahi à présent par l'altemance mangerlêtre mangé, mise en place dans le microcosme familial dès la première scène. Dans ce discours, Shakespeare radicalise le topos biblique et homilétique des gros poissons qui mangent les petits, allant même au-delà de l'ambivalence naissante que l'on décèle dans le tableau de Breughel, actuellement au Musée Boymans de Rotterdam, Les gros poissons mangent les petits. Dans Lear, on en est arrivé au nec plus ultra du cannibalisme réciproque, homophagie tous azimuts sans distinction de taille.

En fait, ce discours d'Albany constitue jusque dans des détails de lexique le pendant symétrique du renversement ogre-nourrisson du premier acte, avec, cette fois, un ordre inversé. En effet les monstres marins qui s'entredévorent constituent le deuxième volet d'un diptyque symétrique, dont le premier représente au contraire l'emblème d'une oralité pleine de tendresse, celle de l'ourse envers l'ourson : $\ll A$ father [...] Whose reverence even the headlugg' d bear would lick, / Most barbarous, most degenerate, you have madded». Ces deux diptyques symétriques, du début et du centre de la pièce, sont les seuls cas dans l'œuvre où surgit l'épithète-limite barbarous, associée chaque fois à la racine generare (generation, ici degenerate). Le chiasme quasi iconique qu'ils constituent donne à voir les termes limites du domaine de l'oralité dans Lear : vision «interne», délirante, du héros tragique, qui se reflète, inversée, dans le miroir «extérieur» du personnage chorique contemplant le cataclysme dans lequel elle s'estréalisée. C'est ce renversement qui, en dernière analyse, joue le rôle a la fois de véritable péripétie (peripeteia) tragique du Roi Lear et de fantasme fondateur de la folie.

Voilà l'un des pôles de cette première scène. Il y a un autre pôle qui est de l'ordre non de la syntaxe mais de la sémantique, non pas l'action de manger ou d'être mangé, cherché ou être cherché, zetôn/zetoumenon dans Edipe Roi, mais la définition exacte de l'objet en question, de ce qu'on est en train de manger. En d'autres termes, il faut être bien clair quant à la nature même de la cérémonie à laquelle on est convié d'assister dans cette première scène, qui a suscité tellement de critiques sur le plan de la sacro-sainte vraisemblance. Et pour cause. Car ce n'est pas de vraisemblance qu'il s'agit mais de vérité du fantasme. Or, à y regarder de plus 
près, les formules employées par le vieux roi pour consacrer la division territoriale prennent des accents pour le moins inattendus. Quelques lignes à peine après notre diptyque, Lear reprend le fil de ses fantasmes : partageant entre ses deux gendres le dernier tiers du royaume, il leur intime l'ordre très exactement de «digérer» cette troisième portion: «With my two daughters dowers digest the third». Si l'on examine de près le texte de Shakespeare, ce que nous contemplons à travers l'encomium ému, plein à la fois de fierté et d'attendrissement que Lear fait de son territoire, l'on s'aperçoit de ce qu'il s'agit en réalité d'une anamorphose, d'un paysage anthropomorphe, genre qui a atteint son apogée à la fin du 16e siècle dans les cuvres du dénommé Maître des Pays-Bas Méridionaux. Ce dont Lear dénombre les avantages, c'est nulle autre que la géante baudlairienne, féconde de corps et ample des hanches, à l'ombre protectrice et bienfaisante : «Of all these bounds, event from this line to this, IWith shadowy rivers and wide-skirted meads, IWe make thee lady». Voilà l'Imago protecteur et bon, nourricier et tendre, que Lear espère communiquer à ses filles en leur donnant à «digérer» / ingérer ce qui en fait est ni plus ni moins qu'un repas totémique inversé, c'est-à-dire maternel. Ce rituel liminaire de la prova d' amore, c'estaussi etsurtout le démembrement, le sparagmos, la digestion de la mère morte par sa progéniture. C'est de là que découle ce déferlement d'images et d'actes que la critique qualifie de «primitifs». C'est aussi cette dimension qui éclaire le véritable sens des tout premiers mots prononcés par Lear quand il apparaitt pour la première fois, en parlant mystérieusement de son «darker purpose», expression dont Onions signale que c'est le premier exemple connu où dark s'emploie de cette manière, et que toute l'ingéniosité de William Empson ne fait qu'effleurer. Cet emploi s'apparente évidemment à l'obscurcissement de la raison, que l'on trouve par exemple dans le «Nosce Teipsum» de Sir John Davies : «But then grew Reason dark, that she no more/Could the fairforms of Good and Truth discern». Mais cela ne suffit pas à rendre compte de ce qu'il y a de spécifiquement - d'étrangement -inquiétant dans cette «darker purpose». Carc'est d'Unheimlichkeit qu'il s'agit, de ce qui, à travers la fiction originale, relève du fantasme originaire. En dernière analyse, ce «dark» liminaire renvoie en fait à l'emploi du même épithète au centre de la pièce, dans l'énoncé oraculaire, chorique, d'Edgar à la fin de l'Acte III, sc. IV : «Child Rowland to the dark tower came», où la gueule du géant cannibale attend le furioso - Orlando as Child - dans sa tour, de toute évidence phallique. Du sujet, Lear est passé à la position objet, mais le noyau reste le même, ainsi que l'ambiguitté sur le plan de la pulsion. A ce propos on peut rappeler que dans The True Chronicle History, le récit contient au tout début une allusion à la mort de l'épouse royale et aux funérailles de celle-ci. Dans la tragédie de Shakespeare, elle reste innommée dans cette première scène. Cependant cette absence n'est que la mesure d'une présence autrement prégnante : elle est omniprésente mais innommable. Symptomatique en est la constellation qui se construit autour de l'épithète «fair» dans cette scène. Il sert à évoquer les charmes du royaume : «this ample third of our fair kingdom», mais il a déjà évoqué ceux de la femme séduite par Gloucester lors de la «saucy» conception de son fils illégitime : 
«Yet was his mother fair». A la fin de la scène, France crée un rapport explicitement spéculaire entre la terre et sa future femme : «Fairest Cordelia... Queen of our fair France». Opulent, épithète par excellence de la sensualité et de la plénitude, s'emploie de la même façon : («A third more opulent than your sisters». L'anamorphose est à fleur de texte.

En fait, si archaïque qu'en soit la formulation, ce fantasme de repas totémique matriarcale rejoint, au moins dans un premier temps, plutôt que l'iconologie germanique avec ses Narrenschiffe et ses Niemand, la tradition italienne comme elle se définit chez l'Arioste et le Tasse, où la Folie n'est pas tant l'outrecuidance de la raison, l'humiliation d'un faux savoir confronté à l'indignitas hominis comme à la vanitas mundi. En Italie, où la nef et sa cohorte sont pratiquement inconnus, la Folie est essentiellement Oubli, je cite Carlo Ossola, «la régression d' une pensée [...] qui veut s' oublier, $[. .]$.$l 'irresponsabilité sublimée en$ innocence ${ }^{15}$, très exactement le projet de nursery que chérissent Lear et 1'Eugène. D'ailleurs, à folie différente, lieux différents : non la mer des confins du monde, mais «l' île oubliée du paradis perdu, le songe-mensonge des "îlesfortunées", le regret et le désir d' un rachat édénique, état de béatitude [...] en deçà du péché d'origine dans le jardin qui gardait l' homme sans fautes ni responsabilités, sans temps ni mémoire» ${ }^{16}$. De ce point de vue, la naissance de la folie de Lear est à l'image de celle d'Erasme, qui a justement l'avantage de bannir la vieillesse et ses maux : «Quod si locum quoque natalem requiritis [...] ego nec in erratica Delo, nec in undoso mari [...] sum edita, sed in ipsis insulia fortunatis, [...] In quibus neque labor, neque senium, neque morbus est ullus». A travers le sparagmos et le repas totémique se profile ainsi la transformation de l'île qu'est son royaume en un petit archipel d'îlots fortunés, où l'on est irresponsable et en même temps innocent. Si problème il y $\mathrm{a}$, c'est qu'entre temps - entre l'innocence perdue et celle retrouvée - le fruit sur l'arbre a eu largement le temps de changer d'aspect. L'objet oral étant de tous à la fois le plus âprement convoité et le plus labile, le plus mutable, la bonne chère - et la bonne mère - se mue en un clin d'œil en pourriture. D'où l'image (alimentaire naturellement) chez l'Arioste du garçon et de la pomme dans l'Orlando Furioso: «come fanciullo che maturo frutto / ripone, e poi si scorda ove è riposto, / e dopo molti giorni è ricondutto / la dove truova a caso il suo deposto, / si maraviglia di vederlo tutto / putrido e guasto, e non come fu posto / e dove amarlo e caro aver solia, / l'odia, sprezza, n' ha schivo, e getta via» (VII, 17). Soit dit en passant que cette logique, ces épithètes s'appliquent admirablement au renversement de ces autres «fruits mûrs» (maturo frutto) que sont les deux filles aînées du roi, surtout si l'on pense au film de Kosintsev, qui a très bien compris cette dimension, à la différence des deux élégantes de Bergman. En l'occurrence, les îles fortunées de Lear ne restent fécondes guère plus longtemps que les Adonidis horticuli, les jardins d'Adonis, qui en représentaient à l'époque, chez Erasme par exemple ${ }^{17}$, la face inverse.

Dans la hiérarchie alimentaire de la société civilisée, le personnage d'Adonis, en effet, fait figure de «double» de Lear, et cela dans son rapport au bléc ${ }^{18}$. Ce n'est pas un hasard si, apres l'errance, le sauvetage de Lear par la bonne fille a 
lieu dans un paysage qui est évoqué en termes quasi écologiques de la «bonne oralité» : non pas sur la lande, mais «in the sustaining corn [...] in the high grown field». C'est-à-dire exactement à mi-chemin entre l'homophagie des profondeurs marines, d'une part, et de l'autre, l'oralité sublimée au point de devenir séraphique de la prison finale : «We two alone will sing like birds i' th'cage : / When thou dost ask me blessing, I'll kneel down, / And ask of thee forgiveness : so we'll live, I And pray, and sing, and tell old tales, and laugh / at gilded butterflies». C'est que, dans son rapport éphémère, transitoire - entre exclusion sociale et réintégration - au "sustaining corn», la position de Lear est effectivement analogue à celle d'Adonis dans le système alimentaire de la civilisation grecque. Car le blé se situe entre l'eau et la putréfaction d'une part, et les cieux et l'incorruptibilité de l'autre, «à égale distance de la bestialité sanguinaire des animaux sauvages se dévorant tout crus les uns les autres et de la pure félicité des Immortels».

en position médiane, et [...] "abonne distance", correspond[ant], du point de vue des grecs, a la vie normale des hommes civilisés, ou s'équilibrent le sec et l' humide et qui constituent une forme de nourriture spécifiquement humaine. Loin d'incarner l' esprit du blé, Adonis s'inscrit tantôt au-deld, tantôt en deçd des céréales. Son destin, qui le mene directement de la myrrhe [pour les dieux] a la laitue [pour les poissons] sert en quelque sorte d'indicatif pour signifier le cour-circuitage des céréales, leur mise entre parenthèses. Il illustre ainsi la tentation et les dangers d' une condition de vie qui prétendrait échapper d la norme».

A la lumière de ce code, on comprend que ce n'est pas un hasard si le personnage d'Adonis est, lui, le fruit d'un inceste fille-père. Or, la pertinence du parallèle avec l'Antiquité ne réside pas seulement dans l'articulation entre le sec et l'humide, oralité «marine» et oralité divine. La grande fête des céréales de l'Antiquité fait explicitement, et de manière assez extraordinaire, le lien avec Lear. On doit rappeler en effet que la cérémonie dite Sacrum Anniversarium Cereris ${ }^{20}$, célébrée chaque année quand le blé est mûr, «the sustaining corn [is] high grown», fête centrée à la fois sur les relations intrafamiliales et le cycle des plantes alimentaires, comporte un tabou linguistique portant exclusivement sur deux termes, qui sévit tout au long des neuf jours de la fête : les deux mots pater et filia y sont rigoureusement prohibés: «Romae cum Cereri sacra fiunt, observatur ne quis patrem aut filiam nominet, quod fructus matrimonii per liberos constets ${ }^{21}$. C'est que ce couple courtcircuite la bonne fertilité, qui se fonde, elle, sur la distance entre générations, comme la proximité entre époux. Bref, dans le parcours alimentaire de Lear, l'étape transitoire dans «the sutaining corn» fixe le moyen terme, aussitôt occulté, entre les deux pôles apolitiques de l'oralité : l'oralité céleste, séraphique de la prison 
paradisiaque de la fin, etl'oralité sauvage, sanguinaire, de cet antichambre de l'enfer qu'est la cour au début de l'œuvre.

Repas totémique et infanticide cannibale, voilà donc les deux faces inverses et complémentaires, passif puis actif - être mangé/manger - de la problématique orale mise en place dès la première scène, reprise d'emblée, cette fois explicitement, la prochaine fois que Lear arrive sur scène : «Let me not stay a jot for dinner : go, get it ready !» Tels en effet sont les tout premiers mots de l'Acte I, sc. IV. ; et si Kent déguisé y trouve faveur auprès du roi, c'est en couronnant la liste de ses qualités par «to eat no fish», où l'oral et le génital sont de nouveau étroitement imbriqués. En réalité, Shakespeare insiste dans cette partie centrale du premier acte - ce dont la mise en scène la plupart du temps ne tient pas compte - sur le fait que l'action se déroule justement à l'heure du dîner. C'est que Goneril est sortie de scène juste avant en lançant l'ordre : «Prepare for dinner». En d'autres termes, à défaut de réaliser ce que la demande comporte d'excessif, de régressif, du moins entend-elle satisfaire au besoin : elle «fera avaler» à son vieux père un bon repas.

Satisfaire au besoin, non à la demande, qui exige au contraire qu'on se garde bien d'y répondre, voilà l'éthique de l'œuvre. Sinon c'est l'abolition de toute distance entre le sujet et l'Autre, la négation de toute séparation, de toute frontière, en d'autres termes, le cannibalisme universel prédit par Albany, confusion des corps jusqu'à ce qu'il n'existe plus qu'un magma indifférencié qui aspire tout ce qui n'en fait pas déjà partie. C'est cela que la castration veut dire dans le domaine de l'oralité. Lear, comme le lui explique le fou «has [...] left nothing $i$ ' th' middles» $(\mathrm{I}, \mathrm{iv}, 195)$; or, le nez, ajoute-t-il, sert précisémentà séparer les deux yeux, tout comme le phallus les générations.

C'est parce que Cordelia s'est d'emblée soustraite à la demande, à la réciprocité, aux rapports spéculaires, se plaçant, elle, sous le signe du besoin et de la distance, qu'elle échappe à la dangereuse dérive vers l'effondrement des limites, au télescopage des corps. C'est que, en dernière analyse, le rituel de la prova d' amore débouche sur les deux versions inverses et complémentaires de la mauvaise distance : le trop grand éloignement (bannissement de Cordelia) et la trop grande proximité (de Lear et de celles en faveur de qui il abdique). Dans le cas d' Édipe Roi, «répondre à la question (de la Sphinge/Jocaste) c'est pratiquer l'inceste et rejoindre le terme trop prochain» ${ }^{2}$. Dans le cas du Roi Lear, répondre à la demande, c'est aussi pratiquer l'inceste mais sous son aspect pré-cedipien, spécifiquement oral ; non pas rejoindre par le sexe le terme trop prochain, mais par la dévoration se l'incorporer, se fondre en lui une fois pour toutes. Le Roi Lear, c'est $C E$ dipe Roi sous sa face kleinienne. Du même coup, c'est aussi, et par là même, une allégorie de la demande, où le «darker purpose» de Lear nous restitue le sens original, originaire, du terme : demandare, se confier soi-même, ses biens et ses besoins à l'autre.

Cette æuvre est ainsi la représentation limite (Urszene, l'extrême étiage de la régression) de la négation des limites, chez celui qui, dans le droit romain, était déjà, comme nous le verrons, «un cas limite».

De ce point de vue, la folie de Lear est très exactement la négation non pas 
tant de la raison mais de la sagesse telle que la définit Pierre Charron dans le premier chapitre de son traité sur le sujet, paru trois ans plus tôt en 1601, et qui constitue peutêtre le meilleur commentaire de l'époque sur l'entreprise d'aliénation à la fois institutionnelle et intellectuelle, voire immobilière, du vieux roi. Dès la première page du livre I, «Qui est de la Cognoissance de soy, et de l' humaine condition», nous lisons en effet: «Aussi voyons-nous que chaque chose pense à soy, s'estudie la premiere, a des limites à ses occupations et désirs [...] Et toy homme, [...] tu t'oublies, tu te respends, et te perds au dehors, tute trahiset te desrobes à toymesme, tu regarde tousjours devant toy, ramasse toy et t' enferme dedans toy : examine toy, espie toy, cognoy toy.»Et le texte de Charron poursuit par des vers latins (de Manilius ?) qui sérient quasiment dans l'ordre les différents aspects etétapes de cette abdication de soi et du sien qu'est, à travers la demande (de prove d'amore), l'acte de de-mandare :

Nosce teipsum, nec te quaesieris extra [ne te cherche pas au dehors] Respue quod non es [phrase clé où l'oralité brutale transparaît clairement. Non pas «ingère ce qui n'est pas toi», mais «recrache - respuere - ce qui n'est pas toi»] tecum habita [Lear, lui, entend habiter partout sauf chez lui, projet qui se termine sur la lande]

Noris quam sit tibi, curta supellex [le bagage/mobilier - supellex - c'est le «train» de Lear, qu'il voit justement se raccourcir (curta) de plus en plus, ce qui renvoità toute la problématique des restes («my rest», Goneril's «remainders»), à la notion de part maudite, part somptuaire, et à l'économie du potlach, que je n'ai pas le temps d'aborder ici, mais qui est capitale).

Tu te consule

Te ipsum concute, [où il faut entendre, en association avec le respue plus haut, non seulement le sens usuel, mais aussi cet emploi secondaire de concutere aliquem : «fais cracher toi-même», c'est-àdire rends à toi ce que tu as donné ou vas donner. En l'occurrence, c'est seulement au début de l'acte III que Lear, par le biais des éléments, va se mettre à (re)cracher «Spit, fire ! Spout, rain!»] nunquid vitiorum

Inseverit olim natura, aut etiam consuetudo mala. [car jamais la nature ne t'aura donné aucun des vices mais plutôt la mauvaise habitude. Lear «hath ever but slenderly known himself») ${ }^{23}$

A travers les codes de la connaissance établis par Charron se découvre, comme dans un miroir, l'image inversée d'une anatomie de la folie de Lear : voici en effet 


\section{l'errance du fou (tecum habita);}

le savoir du fou - la quête de soi dans le miroir déformant de l'autre, renversée à la fin en quête de l'autre, dans le miroir au mirage mortel («Look there, look there». Dies);

enfin l'économie du fou - la dépense plutôt que la réserve, que lui enseigne The Fool («Have more than thou showest, /Speak less than thou knowest». etc.), mais aussi et surtout l'économie du fou telle qu'elle était prévue dans le droit romain. Il est piquant à ce propos de rappeler que dans le code hérité de Justinien (Digeste 27 et 10, Institutes I et 22, articles ranimés dès le début du 14 e siècle) ${ }^{24}$, le fou n'était pas seul à occuper la catégorie juridique conçue à son intention. Il la partageait, en effet, avec un autre compère : très exactement celui qui, pour emprunter l'expression de Charron, «se perd dehors», non seulement sur le plan du savoir ou de l'affect mais sur le plan économique, c'est-à-dire celui qui aliène ses biens contre son propre intérêt et contre celui de ses dépendants ou descendants, le dénommé «prodigus et bonorum suorum dissipator». A l'un comme à l'autre était destinée l'institution de droit civil qu'était la curatelle, en la personne du tutor mente capto. Ce n'est qu'au $17 \mathrm{e}$ siècle, avec l'enfermement, que le droit civil introduira la distinction - qui reste inconnue dans le droit canon - entre le furiosus et le bonorum suorum dissipator. Ce qui se passe de commentaire dans le cas qui nous occupe. Ce sont pour ainsi dire les deux moitiés d'un même fou. Ou, comme le dit Charron lui-même un peu plus loin (ch. 15), «La sagesse et la folie sont fort voisines. Il n'y a qu' un demi-fou de l' un a l'autre. Cela se voit aux actions des hommes insensés» (nous soulignons).

Il n'en reste pas moins que ce que Charron nous dévoile surtout à travers ces vers liminaires, c'est, par le biais des deux verbes les plus marqués sémantiquement, à savoir respuere et concutere, la double face symétrique de l'oralité folle qui sous-tend, dans l'acte de de-mandare (d'aliéner ses biens) cette économie archaïque proche du potlach : respectivement respue quod non es, éloigne le trop près, vomisle, et Te ipsum concute, rapproche le trop lointain, c'est-à-dire ce que tu te dois à toimême. Et Charron de conclure, en français, par une phrase qui renverse explicitement le logos spéculaire de la prova d' amore : «et voyld justice et paixpartout.Bref, nous n'avons point de plus beau miroir et de meilleur livre que nous mesmes». A cet égard, Lear est non seulement une mise à nu de l'oralité dans ce qu'elle a de plus sombrement (darkly) archaïque : cette mise à nu est en même temps - et en toute logique - une mise en scène paradigmatique de la méconnaissance telle que celle-ci s' articule dans le stage dit «du miroir , celui qui se place justement sous le signe de la toute puissance de la pulsion orale. 


\section{NOTES}

i Toutes références à l'édition Arden, Methuen, London, (1952) 1968

1 Sir Philip Sidney, The Defense of Poesy, in Selected Poetry and Prose, ed. Craik, London, Methuen, (1965), 1967, p. 33.

2 Op.cit., p. 25

3 Op.cit., p. 35.

4 Dans Essais de Psychanalyse Appliquée, Paris, Gallimard, 1933, notamment p. 9-12.

5 Paris, Gallimard, Pléiade, ed. Robin, IX, 571-2, p. 1174.

6 In The Wheel of Fire, London, Methuen (1930), 1968, p. 182 (our italics).

7 Cette ambivalence de registre s'éclaire enfin, si besoin était, de la comparaison avec le discours de celui qui, dans l'œuvre de Shakespeare, fait figure de première ébauche de Lear, le Duc de Milan dans Two Gentlemen of Verona. Ce dernier, alors qu'il prononce à l'égard de sa fille un décret de bannissement quasi identique, nous donne à entendre une première version de ce que l'on trouvera plus tard dans la bouche de Lear - à une exception près, et qui est capitale : «this pride of hers, / Upon advice, hath drawn my love from her ; I And, where I thought the remnant of mine age I Should have been cherished by her child-like duty, I I am now full resolved to take a wife, I And turn her out to who will take her in» (III, i). face œdipienne ici, face pré-olympienne dans Lear.

8 C'est elle, plus encore que Freud, qui s'est penchée sur ces stades archaïques de la structuration de la personnalité, notamment dans les essais cliniques contenus dans Essais de Psychanalyse, Paris, Payot, (1921-45), 1974.

9 Euvres, Paris, Lemerre, 1868, vol. I, p. 18-9.

10 Cf. dans La Folie et le Corps, Paris, Presses de l'E.N.S. Ulm, le très bel article de Frank Lestringant, «Rage, fureur, folie cannibales : le Scythe et le Brésilien» p. 49 sq. Op.cit., p. 70.

11 Lestringant, op. cit. p. 78.

12 Cité dans «Rage, fureur, folie».

13 En effet, le vers de Jodelle, «Biens, ie dy, que iamais n'acquirent / Les parents qui naistre me firent» peut aussi être entendu dans le sens «Bien, je dis, que jamais nacquirent les parents...».

14 The Structure of Complex Words, London, 1951, p. 127.

15 Dans «Métaphore et inventaire de la folie dans la littérature italienne du XVIe siècle», Folie et Déraison à la Renaissance, Actes d'un colloque du même titre tenu à Bruxelles en 1973, Bruxelles, Presses de l'Université, 1976, p. 171.

16 Op.cit.

17 Cf. Morias Encomium, Basle, in aedibus Io, Frobenii, 1515, pp. 77-8. 
18 Orlando Furioso, VII, 71.

19 Jean-Pierre Vernant, dans Introduction à Les Jardins d'Adonis, Paris, Gallimard, 1972, pp. v-vi. (nous soulignons).

20 Marcel Detienne, Les Jardins d'Adonis, op. cit. p. 149-50.

21 Op.cit. p. 150. Il s'agit d'une citation de Servius Danielis dans son commentaire de Virgile, In Virg. Aen. IV, 58.

22 Catherine Clément, Vie et Légendes de Jacques Lacan, Paris, Grasset, 1981, p. 133.

23 De la Sagesse, Paris, Bastien, 1783, p. 1-3.

24 Cités par Paul Forier dans Folie et Déraison, op. cit., p. 30-33. 